\title{
Electroluminescence and photoluminescence studies on carrier radiative and nonradiative recombinations in metal-oxide-silicon tunneling diodes
}

\author{
Miin-Jang Chen, Jui-Fen Chang, Jia-Liang Yen, and Chen S. Tsai ${ }^{\text {a) }}$ \\ Institute for Applied Science and Engineering Research, Academia Sinica, Taiwan, Republic of China \\ Eih-Zhe Liang and Ching-Fuh Lin \\ Graduate Institute of Electro-Optical Engineering, National Taiwan University, Taiwan, Republic of China \\ C. W. Liu \\ Department of Electrical Engineering, National Taiwan University Taiwan, Republic of China
}

(Received 12 August 2002; accepted 3 January 2003)

\begin{abstract}
Electroluminescence (EL) and photoluminescence (PL) measurements at silicon band gap energy from metal-oxide-semiconductor tunneling diodes on silicon have been carried out to study the carrier radiative recombination and nonradiative recombination near the silicon/silicon dioxide $\left(\mathrm{Si} / \mathrm{SiO}_{2}\right)$ interface. The temporal EL response indicates that the radiative recombination coefficient involved is as much as ten times greater than that of the bulk silicon. However, the nonradiative recombination is still the dominant carrier recombination process. Voltage-dependent PL shows that PL intensity increases with the bias voltage. This voltage dependence is attributed to the variations of nonradiative recombination rates due to the change of Fermi level with the bias voltage. The intensity of EL is found to be less temperature dependent than that of PL. The near-band-edge Fermi level for EL leads to the reduced and less thermal-active nonradiative recombination as compared to PL. This study shows that Fermi level near the $\mathrm{Si} / \mathrm{SiO}_{2}$ interface strongly influences the nonradiative recombination rates and the resulting EL and PL intensities. (C) 2003 American Institute of Physics. [DOI: 10.1063/1.1556182]
\end{abstract}

\section{INTRODUCTION}

Because of the dominance of silicon in ultralarge-scaleintegration (ULSI) circuits, there has been a strong desire to realize monolithic integration of microelectronics and optoelectronics in a single silicon chip. Unfortunately, such monolithic integration has encountered great difficulty due to indirect band gap of silicon that results in inefficiency as light sources. One approach to overcome the inefficiency is to simultaneously enhance carrier radiative recombination and reduce the associated nonradiative recombination. Thus, it is important to study the carrier radiative and nonradiative recombinations involved in silicon.

On the other hand, when the dimensions of metal-oxidesemiconductor field-effect-transistor (MOSFET) are scaled down to deep submicrometer in the state-of-the-art ULSI circuits, the thickness of gate oxide is in the range of $15-30 \AA .{ }^{1}$ Characterization of the metal-oxide-semiconductor (MOS) devices with such thin gate oxides requires accurate determination of the material parameters, such as the minority carrier lifetime, to provide the information about the low defect densities near the $\mathrm{Si} / \mathrm{SiO}_{2}$ interface in the present ULSI devices. Conventional methods such as the high/low-frequency capacitance-voltage analysis, conductance method, chargepumping method, and transient capacitance-time Zerbst analysis, etc., have been successfully used to measure the

\footnotetext{
a) Author to whom correspondence should be addressed; electronic mail: cstsai@phys.sinica.edu.tw
}

minority carrier lifetime and interface state density $\left(D_{\mathrm{it}}\right)$ of the MOS devices with relatively thick gate oxide. ${ }^{2-4}$ However, as the thickness of the gate oxide shrinks, the leakage current tunneling through the ultrathin gate oxide increases significantly and the MOS devices result in tunneling diodes. Therefore, the significant series resistance in the MOS capacitors due to the ultrathin oxide complicates the analysis and the modeling based on the above conventional measurement methods becomes invalid. ${ }^{5}$ Clearly, a simple method needs to be developed for characterization of the MOS devices when the tunneling current through the gate oxide becomes significant.

In this article, we show that the electroluminescence (EL) and photoluminescence (PL) measurements at silicon band gap energy from MOS tunneling diodes on silicon provide a convenient way to optically probe the electrical and optical properties of the $\mathrm{Si} / \mathrm{SiO}_{2}$ interface. The carrier radiative and nonradiative recombinations in the MOS tunneling diodes are investigated from the temporal response of EL, voltage-dependent PL intensity, and temperature dependence of EL and PL intensities. By measuring the EL signals in the time domain from the MOS tunneling diodes under the injection of a square current pulse, the minority carrier lifetime near the $\mathrm{Si} / \mathrm{SiO}_{2}$ interface is extracted. Subsequently, the related information such as the carrier radiative and nonradiative recombination coefficients is derived. Finally, the temperature dependent EL and PL intensities and the voltagedependent PL intensity are also used to characterize the 


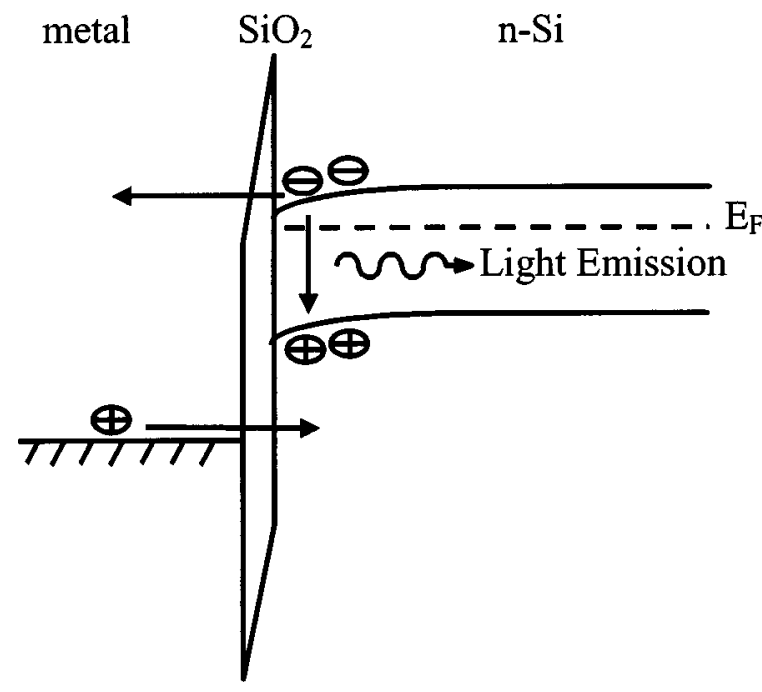

FIG. 1. Schematic band diagram of the MOS tunneling diode.

underlying radiative and nonradiative recombination processes.

\section{DEVICE FABRICATION AND CHARACTERISTICS}

In this work, the ultrathin gate oxide of the MOS tunneling diode was grown by rapid thermal oxidation on silicon substrate. The resistivities of silicon wafers used are 1-10 $\Omega \mathrm{cm}$ for the $n$-type substrate and $1-5 \Omega \mathrm{cm}$ for the $p$-type substrate. Prior to oxidation, the silicon substrate was cleaned by a HF dip. An in situ hydrogen bake was performed before the growth of oxide. After hydrogen bake, ultrathin gate oxide was grown at $900-1000{ }^{\circ} \mathrm{C}$. The gas flows were set at $500 \mathrm{sccm}$ nitrogen and $500 \mathrm{sccm}$ oxygen at a reduced pressure. The resulting ultrathin oxide was then in situ annealed. The temperature was measured by a pyrometer with a close loop control. The thickness of the oxide was measured by ellipsometry and confirmed by high-frequency capacitance-voltage measurements. The measured oxide thickness was in the range of 15-35 $\AA$, thin enough to allow significant carrier tunneling. Aluminum or transparent indium tin oxide (ITO) was subsequently deposited on top of the oxide as the gate electrode. Finally, aluminum was deposited on the backside of the silicon substrate as another electrode of the MOS diode.

Figure 1 depicts the schematic band diagram of the MOS tunneling diode on $n$-type silicon when EL takes place. Under a positive gate voltage, an accumulation layer of majority electrons is formed at the $\mathrm{Si} / \mathrm{SiO}_{2}$ interface. The electrons tunnel from silicon to the gate electrode and the holes tunnel from the gate electrode to silicon. Because of the larger tunneling barrier for hole, the resulting hole tunneling current is much smaller than the electron tunneling current. The tunneling holes recombine with the electrons accumulated at the $\mathrm{Si} / \mathrm{SiO}_{2}$ interface and result in the radiative EL. It is to be noted that even though the electron tunneling current dominates in the total current and acts as the leakage current for radiative recombination, EL is still observed at room temperature.

Figure 2 shows the EL spectra from $\mathrm{ITO}_{\mathrm{SiO}} / n-\mathrm{Si}$

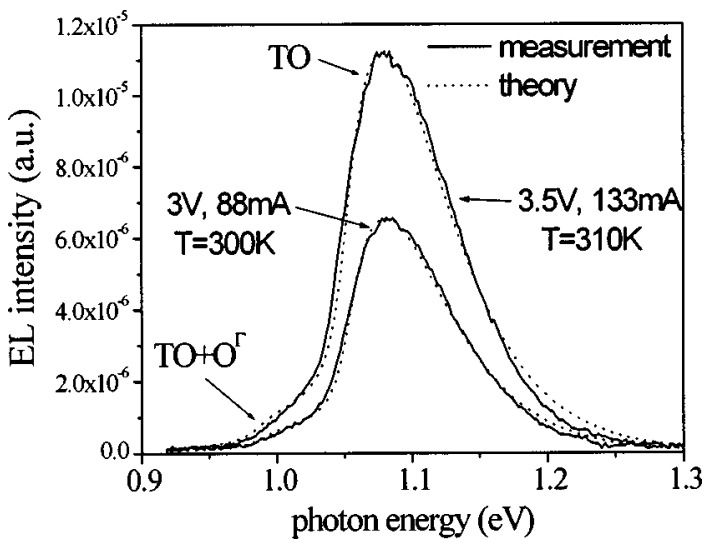

FIG. 2. Room-temperature EL spectra from ITO/33 $\AA \mathrm{SiO}_{2} / n$-Si tunneling diode as well as the theoretical fitting.

MOS tunneling diode on silicon under continuous-wave operation at room temperature. A computer-controlled 1/2 M monochromator together with the conventional lock-in technique were used to measure the emission spectra. An InGaAs $p-i-n$ diode with responsivity of $0.7 \mathrm{~A} / \mathrm{W}$ at $1.08 \mathrm{eV}$ photon energy was placed at the exit of the monochromator to detect the optical signal. The peaks of the spectra are seen to occur at around $1.08 \mathrm{eV}$, which is slightly below the band gap energy of silicon crystal at room temperature $(1.12 \mathrm{eV})$. This energy shift suggests the participation of transverse optical (TO) phonon in the radiative recombination. ${ }^{6,7}$ The lowenergy tail in Fig. 2 is due to the two-phonon process in the radiative recombination. The two-phonon process involves the emission of a momentum-conserving TO phonon and a zone center $\left(\mathrm{O}^{\Gamma}\right)$ phonon. The dashed lines are the calculated spectra in terms of the theoretical model of phonon-assisted and exciton-involved radiative recombination. ${ }^{7}$ Figure 2 shows an excellent agreement between the theoretical spectra and the measured data. The model demonstrates that the optical properties of silicon crystal are strongly influenced by both excitons and phonons. Before recombination, an electron and a hole are bounded to each other under the Coulomb attraction to form an exciton first, and then recombine to emit a photon and a phonon.

\section{TEMPORAL EL RESPONSE}

The temporal response of EL from the MOS tunneling diode under the injection of a square-wave current pulse was measured using scanning-gate integrator and boxcar averager system. ${ }^{8}$ Figure $3(\mathrm{a})$ is the waveform of the injection current with $150 \mu$ s duration and Fig. 3(b) is the measured temporal EL waveforms from the $\mathrm{Al} / \mathrm{SiO}_{2} / n$-Si MOS tunneling diode at a set of injection currents. These measured EL waveforms in the time domain should track the variation of the excess minority carrier density with time. ${ }^{4}$ Therefore, the minority carrier lifetime near the $\mathrm{Si} / \mathrm{SiO}_{2}$ interface can be determined from either the rising-edge or the falling-edge of EL waveform in the time domain. The rising and falling edges of the temporal EL waveforms are fitted using the following expression: 

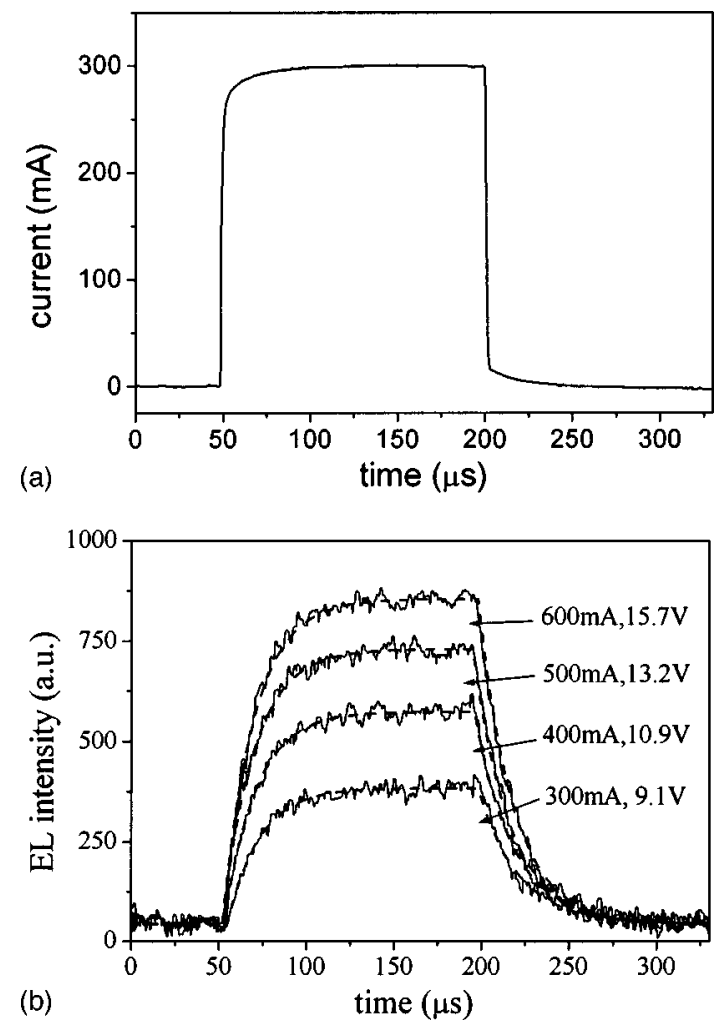

FIG. 3. (a) The waveform of injection current with duration $150 \mu$ s. (b) Temporal EL waveforms under the injection of a square current pulse at various injection currents.

$$
L(t)=L(\infty)-[L(\infty)-L(0)] \cdot \exp \left(-\frac{t}{\tau}\right),
$$

where $L(\infty)$ and $L(0)$ denote the final and initial values of the light intensities, respectively, and $\tau$ is the minority carrier lifetime. The minority carrier lifetime $\tau$ is related to the carrier recombination coefficients $A, B$, and $C$ through the following expression: ${ }^{4}$

$$
\frac{1}{\tau}=A+B \cdot N+C \cdot N^{2},
$$

where $A$ is the coefficient of Shockley-Read-Hall (SRH) nonradiative recombination through the intraband states near the $\mathrm{Si} / \mathrm{SiO}_{2}$ interface, $B$ and $C$, respectively, are the band-toband radiative recombination and Auger recombination coefficients, and $N$ is the majority carrier density. Figure 3(b) shows a good match between the experimental data and the fitting curves. The carrier recombination rates $1 / \tau$ extracted from the curve fitting in Fig. 3(b) at various injection current densities are shown in Fig. 4.

It is seen that at the rising edge of EL waveform, the recombination rate is independent of the injection current. According to Eq. (2), this indicates that the SRH recombination is the dominant carrier recombination process. On the other hand, the recombination rate increases with the injection current at the falling edges of the EL signal, which means that the effect of radiative and Auger recombination cannot be neglected at the EL falling edge. Using least squared method, it is shown that a parabolic curve can well fit the data points of EL falling edge. In the Appendix, we

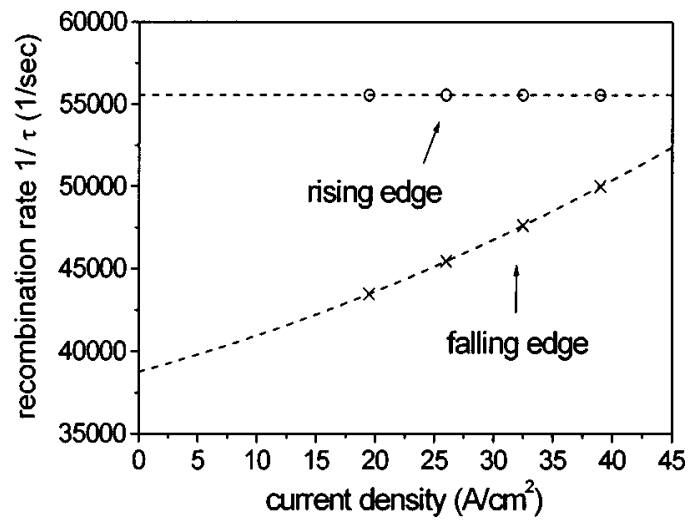

FIG. 4. The carrier recombination rates $1 / \tau$ vs the injected current level at the EL rising and falling edges.

show that it is a valid assumption that the majority carrier density $N$ is proportional to the injection current density $J$, i.e., $N=k J$, when the applied gate voltage is greater than 7.5 $\mathrm{V}$ for an $\mathrm{Al} / \mathrm{SiO}_{2} / n$-Si MOS tunneling diode with $30 \AA$ gate oxide. Equation (2) then results in the following parabolic relation between the carrier recombination rate $1 / \tau$ and the current density $J$ :

$$
\frac{1}{\tau}=A+B \cdot k J+C \cdot k^{2} J^{2} .
$$

Therefore, the data shown in Fig. 4 reveal the relative amounts among the SRH recombination, radiative recombination, and Auger recombination.

The intercept $A$ of the parabolic curve [Eq. (3)] obtained from the least squares fitting to the data points of EL falling edges in Fig. 4 is the SHR recombination coefficient. The SHR recombination lifetimes $\tau_{\mathrm{SHR}}\left(\tau_{\mathrm{SHR}}=1 / A\right)$ at the rising and falling edges are determined to be 18.0 and $25.8 \mu \mathrm{s}$, respectively. It is well known that the $\mathrm{Si} / \mathrm{SiO}_{2}$ interface states with energies near the Fermi level constitute the most active SRH recombination centers ${ }^{2-4}$ and the SRH recombination rate approaches a maximum when the recombination centers are located near the midgap region. ${ }^{4}$ The difference in SHR recombination rates between the rising edge and the falling edge of EL signal could be due to a difference in the positions of Fermi level. As the current is just turned on (rising edge), the MOS tunneling diode is still near the flatband condition and the position of Fermi level is close to the midgap. Thus the SRH recombination rate is large and depends less on the carrier density $N$ because there is no large accumulation of carriers near the $\mathrm{Si} / \mathrm{SiO}_{2}$ interface. However, when the MOS tunneling diode is operated at the accumulation region, the Fermi level in the $n$-type silicon substrate is located near the conduction band edge and its variation with the applied voltage is small. There is little change in the SRH recombination rate. Therefore, the position of Fermi level is far away from the valence band edge at the initial stage of EL falling edge. The value of SRH recombination rate $A$ is reduced and is almost the same at various injection current levels at the EL falling edge. As a result, there is lower SRH recombination rate at the EL falling edge when compared to the rising edge. The near-band-edge Fermi level also results 


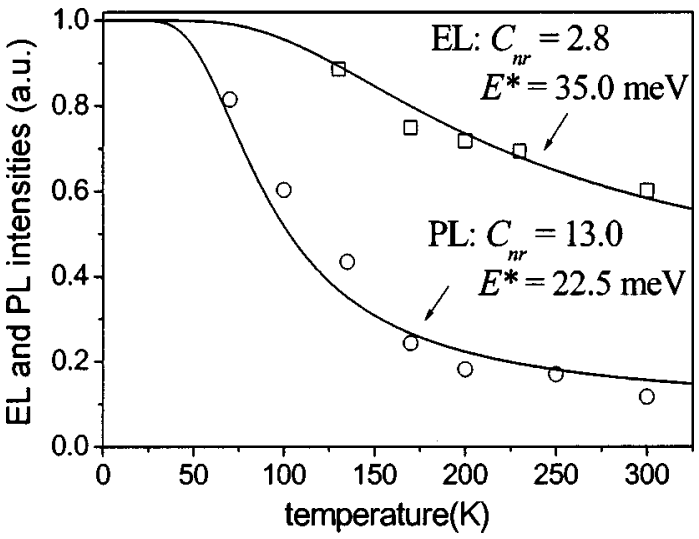

FIG. 5. The dependence of EL and PL intensities on temperature. The squares and circles are the data points of EL and PL experiments, respectively. Solid line: theoretical fitting.

in a very large amount of majority carrier accumulated at the $\mathrm{Si} / \mathrm{SiO}_{2}$ interface. This large majority carrier accumulation leads to the increase of the recombination rate with the injection current at the EL falling edge, as depicted in Fig. 4.

The least square fitting using the parabolic curve to the data points of EL falling edges also reveals the information about the radiative and Auger recombinations. The values of $B \cdot k$ and $C \cdot k^{2}$ are determined to be 194.6 and $2.4 \mathrm{~cm}^{4} / \mathrm{A}^{2} \mathrm{~s}$, respectively. Thus, the ratio among the components of SRH, radiative, and Auger recombinations is 1:0.196:0.096 at the injection current density of $39 \mathrm{~A} / \mathrm{cm}^{2}$. For the falling edge, the SRH recombination is still the most important carrier recombination process, but the contributions from radiative and Auger recombinations cannot be ignored. If the Auger recombination coefficient $C$ of $10^{-31} \mathrm{~cm}^{6} / \mathrm{s}$ for holes in bulk silicon is used, ${ }^{4}$ the value of coefficient $B$ is determined to be $4 \times 10^{-14} \mathrm{~cm}^{3} / \mathrm{s}$. This value is nearly ten times larger than that of bulk silicon. ${ }^{4}$ This large increase in the radiative recombination coefficient in silicon may possibly be due to the momentum compensation facilitated by the $\mathrm{Si} / \mathrm{SO}_{2}$ interface roughness and the localized carriers in the potential well near the $\mathrm{Si} / \mathrm{SiO}_{2}$ interface. $^{9-11}$ Therefore, the larger value of $B$ coefficient facilitates measurement of the minority carrier lifetime near the $\mathrm{Si} / \mathrm{SiO}_{2}$ interface using the temporal $\mathrm{EL}$ response. The information related to the quality of $\mathrm{Si} / \mathrm{SiO}_{2}$ interface can be further deduced based on the measured minority carrier lifetime to characterize the MOS devices with ultrathin gate oxides.

\section{TEMPERATURE DEPENDENCE OF EL AND PL INTENSITIES}

Figure 5 shows the measured temperature dependence of the EL and PL intensities from $n$-type silicon. Both luminescences are seen to be quenched by temperature. However, the data show that the EL intensity of the MOS tunneling diode exhibits weaker temperature dependence, as compared to PL. For EL, the normalized luminescence intensity decreases to about 0.6 when the temperature rises to $300 \mathrm{~K}$. In comparison, the normalized PL luminescence drops to less than 0.1 as the temperature increases to $300 \mathrm{~K}$. The excess carriers for EL and PL are generated by either tunneling effect or photo- excitation. Since the amounts of the carrier injected by both methods are insignificantly dependent on the temperature, the difference in temperature dependence for EL and PL should not be due to the method of carrier generation. Nevertheless, these excess carriers will eventually vanish through either radiative or nonradiative recombination. It is well known that increase in temperature would allow the onset of nonradiative recombination process. ${ }^{12}$ As a result, the difference in temperature dependence between EL and PL intensities may be attributable to the difference in the nonradiative recombination processes.

Nonradiative recombination states induce a perturbation in the band structure and produce a barrier height $E^{*}$ around the recombination center. ${ }^{12}$ The energy $E^{*}$ is called the activation energy of nonradiative recombination states. Only the carriers with sufficient energy to overcome this barrier can recombine nonradiatively. Therefore, increasing the temperature will enhance the nonradiative recombination and thus quench the luminescence. The usual approach to extract the activation energy $E^{*}$ from the temperature dependence of luminescence intensity is to fit the experimental data by the following theoretical and semiempirical expression: ${ }^{12-15}$

$$
\eta(T)=\frac{1}{1+C_{\mathrm{nr}} \exp \left(-E^{*} / k_{B} T\right)},
$$

where $\eta$ is the luminescence intensity normalized at $0 \mathrm{~K}, T$ is the temperature, $k_{B}$ is the Boltzmann constant, and $C_{\mathrm{nr}}$ is a constant describing how efficiently the nonradiative recombination state reduces the luminescence. An efficient nonradiative recombination process corresponds to a large value of $C_{\mathrm{nr}}$ and a small value of activation energy $E^{*}$.

The values of $C_{\mathrm{nr}}$ and $E^{*}$ obtained by curve fitting to the experimental PL and EL data are also shown in Fig. 5. For $\mathrm{EL}$, there is a smaller $C_{\mathrm{nr}}$ value (2.8 vs 13.0 for PL) and a larger $E^{*}$ value (35.0 vs $22.5 \mathrm{meV}$ for PL). This indicates that the nonradiative recombination state is less thermally active and less efficient, which results in a greater luminescent efficiency for EL at room temperature. For the MOS tunneling diode operated in the accumulation region, the applied gate voltage attracts the majority carriers and causes their confinement in a small potential well near the $\mathrm{Si} / \mathrm{SiO}_{2}$ interface. On the other hand, the excess carriers generated by the laser illumination distribute over about one carrier diffusion length. Since the PL intensity is known to be very sensitive to the nonradiative recombination on the surface, ${ }^{4}$ the nonradiative recombination events in the bulk silicon can be neglected. Therefore, the difference in the nonradiative recombination rate due to the different Fermi-level positions near the $\mathrm{Si} / \mathrm{SiO}_{2}$ interface for EL and PL is the most likely cause for the significant difference in the temperature dependence of EL and PL shown in Fig. 5.

\section{VOLTAGE-DEPENDENT PL INTENSITY}

To further confirm the arguments stated above, voltagedependent PL intensity was also measured from an $\mathrm{ITO} / \mathrm{SiO}_{2} / p$-Si MOS tunneling diode at room temperature. The transparent ITO electrode allows the transmission of $514.5 \mathrm{~nm}$ green line from an $\mathrm{Ar}^{+}$laser for optical pumping 


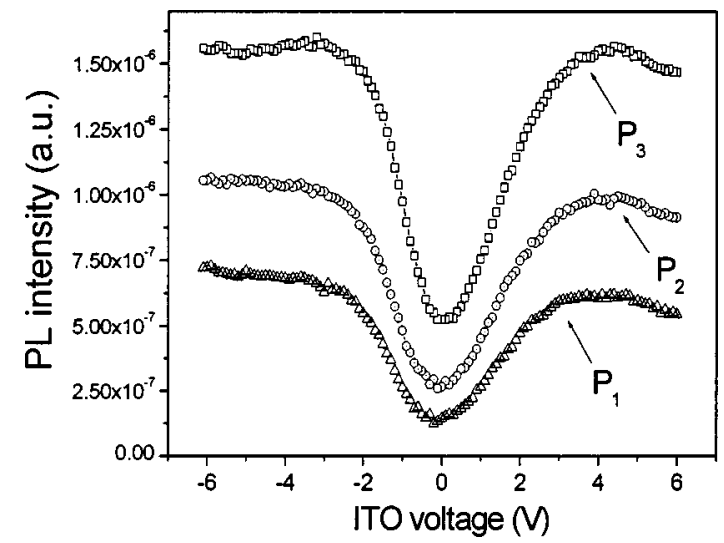

FIG. 6. The dependence of PL intensity on gate bias voltage at various optical pumping power $\left(P_{3}>P_{2}>P_{1}\right)$.

and simultaneous application of the bias voltage. Figure 6 shows the variation of PL intensity with the applied gate voltage at different pumping powers. When the MOS tunneling diode was not biased, the measured PL was similar to the case of bulk silicon. However, the luminescence intensity was found to increase no matter when the device was under forward or reversed bias. In Fig. 6, the negative and positive gate voltages correspond, respectively, to the forward and reverse bias of the MOS tunneling diode on $p$-type silicon substrate. For the devices using the $n$-type silicon substrate, the measured results of voltage-dependent PL intensity were similar. Because the PL was measured only at the chopped frequency of pumping laser, the measured PL intensity did not contain the possible EL signal. Figure 7 shows the normalized PL spectra at the gate voltage of $-4,0$ and $+4 \mathrm{~V}$, respectively. Though the PL intensity increases with the applied gate voltage, Fig. 7 shows that the spectral shapes remain the same. This means that the carrier radiative recombination mechanism is not strongly influenced by the applied gate voltage. The current-voltage $(I-V)$ characteristics of the MOS tunneling diode with and without the optical pumping are shown in Fig. 8. At reverse bias, the photogenerated carriers in the silicon substrate diffuse and are swept by the bias electric field to the $\mathrm{Si} / \mathrm{SiO}_{2}$ interface, and then tunnel through the oxide to the ITO gate. Therefore, the tunneling

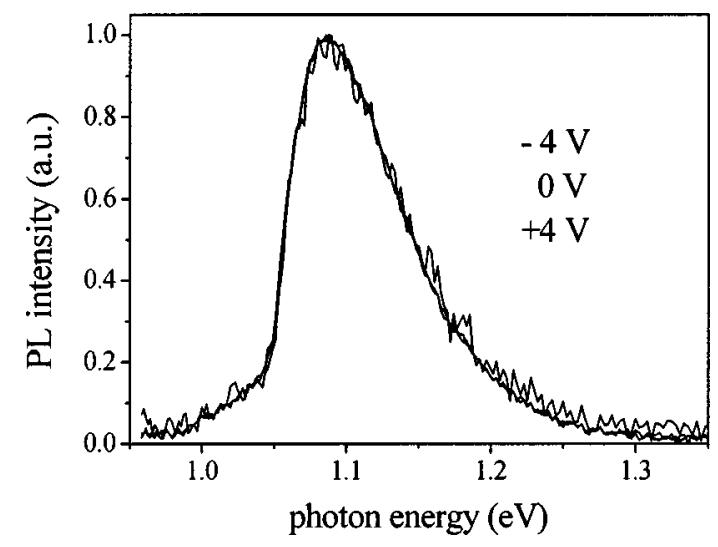

FIG. 7. The normalized PL spectra under the applied gate voltage $-4,0$, and $+4 \mathrm{~V}$, respectively.

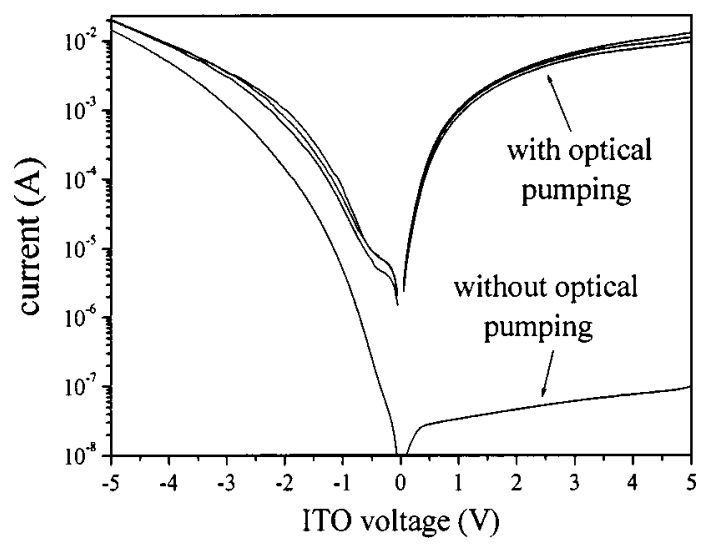

FIG. 8. The $I-V$ characteristics of the ITO/33 $\AA \mathrm{SiO}_{2} / p$-Si tunneling diode with and without the $\mathrm{Ag}^{+}$laser pumping.

current increases significantly at reverse bias under laser illumination. Clearly, an $\mathrm{ITO} / \mathrm{SiO}_{2} / \mathrm{Si}$ structure with the ultrathin oxide could be utilized as a photodetector.

Several important features are revealed by the measured data shown in Fig. 6. The PL intensity remains low for a small bias voltage between 0 and $-0.8 \mathrm{~V}$. In this range of bias voltage, the Fermi level in silicon is near the midgap region and far away from both the conduction and the valence edges, leading to less accumulation of carriers at the $\mathrm{Si} / \mathrm{SiO}_{2}$ interface. From Eq. (2), a low accumulated carrier density results in a small radiative recombination rate. Also, according to the previous PL study, the PL intensity is very sensitive to the surface recombination. ${ }^{4}$ A carefully prepared surface would reduce the surface recombination and, thus, improve the luminescence efficiency. As mentioned in Sec. III, the most active SRH recombination centers are those $\mathrm{Si} / \mathrm{SiO}_{2}$ interface states with energies near the Fermi level. ${ }^{2-4}$ The most effective SRH recombination centers are those located near the middle of the band gap. ${ }^{4}$ Therefore, the nearmidgap Fermi level also results in a large nonradiative recombination rate. The reduced radiative recombination rate and the enhanced SRH recombination due to the nearmidgap Fermi level cause the lower PL efficiency at such a small bias region.

When the forward bias is increased from 0 to $-4 \mathrm{~V}$, the PL intensity increases drastically. Increased forward bias causes bending of the valence band edge toward the Fermi level in the $p$-type silicon substrate and results in accumulation of majority holes at the $\mathrm{Si} / \mathrm{SiO}_{2}$ interface. The large amount of majority holes enhances the radiative recombination rate, and in the mean time the near-band-edge Fermi level reduces the SRH recombination process. Thus, the PL intensity increases with the forward bias voltage. At sufficiently large forward bias voltage, the position of Fermi level near the $\mathrm{Si} / \mathrm{SiO}_{2}$ interface approaches the valence band edge and its variation with the applied voltage is small. This results in a small change for the SRH recombination. Since $\mathrm{SRH}$ recombination near the $\mathrm{Si} / \mathrm{SiO}_{2}$ interface is shown to be the most important carrier recombination process (see Sec. III), the PL intensity eventually saturates under forward bias greater than $-4 \mathrm{~V}$, as shown in Fig. 6 . 


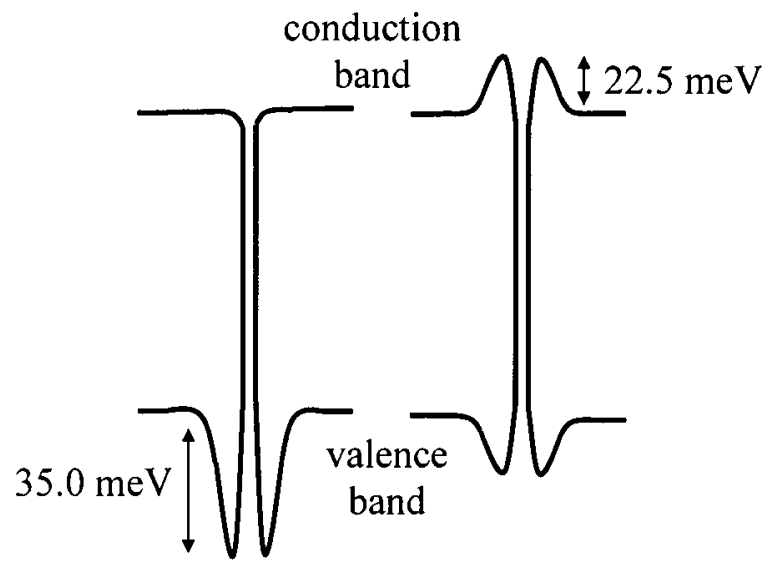

(a)

(b)

FIG. 9. Schematic diagram of nonradiative recombination states for the case of (a) EL and (b) PL.

Similarly, the PL intensity also increases drastically when the reverse bias is increased from 0 to $3 \mathrm{~V}$. The argument provided above for the increase of PL intensity with forward bias also holds for the case of reverse bias. The Fermi level moves toward the conduction band edge and causes reduction of SRH recombination and accumulation of electrons. As a result, the luminescence is greatly enhanced. When the device is under a reversed bias larger than $3 \mathrm{~V}$, the PL begins to drop. This dependence could be attributed to the strong electric field near the $\mathrm{Si} / \mathrm{SiO}_{2}$ interface. Because the silicon surface is inverted and a depletion region is formed under large reversed bias, there exists a strong electric field near the $\mathrm{Si} / \mathrm{SiO}_{2}$ interface. The typical value of ionization field for excitons in semiconductors is in the order $10^{4} \mathrm{~V} / \mathrm{cm} .{ }^{16,17}$ Therefore, the strong electric field $\left(\sim 10^{5} \mathrm{~V} / \mathrm{cm}\right)$ resulting from the $3 \mathrm{~V}$ reversed bias causes the dissociation of excitons and also sweeps the carriers out of the interface before they recombine.

In summary, when the MOS tunneling diode is under either forward or reversed bias, the resulting band bending leads to the near-band-edge Fermi level at the $\mathrm{Si} / \mathrm{SiO}_{2}$ interface. This results in the reduction of the nonradiative recombination through the interface states and also the enhancement of the radiative recombination. Since the SRH recombination is the dominant recombination process in the MOS tunneling diode, the variations of PL intensity with bias voltage as shown in Fig. 6 are mainly the result of the change of SRH recombination rate due to the different position of Fermi level. Therefore, it is concluded that the nearband-edge Fermi level is essential for enhanced luminescence no matter whether the carriers are photoexcited or injected through tunneling. In the case of EL from the MOS tunneling diode on $n$-type silicon substrate, the Fermi level at the $\mathrm{Si} / \mathrm{SiO}_{2}$ interface is near the conduction band. The most active SHR recombination centers are efficient electrons traps, but it is difficult for them to capture the minority holes. Hence Fig. 9(a) is proposed for the schematic diagram of the nonradiative recombination states for EL. There is a large barrier height for the minority holes. In comparison, in the case of PL without bias voltage, the Fermi level is located near the middle of the band gap and leads to an efficient nonradiative recombination process. Similarly, Fig. 9(b) is proposed for the schematic diagram of the nonradiative recombination centers for PL without bias voltage. The barrier height for both electrons and holes is small as compared to that for holes in Fig. 9(a). The nonradiative recombination process is less efficient and less thermally active for EL as compared to PL without bias voltage. Therefore, the value of $C_{\mathrm{nr}}$ is smaller and activation energy $E^{*}$ is larger in the case of EL. The different position of the Fermi level near the $\mathrm{Si} / \mathrm{SiO}_{2}$ interface well explains the difference in temperature dependence of EL and PL intensities from silicon.

\section{CONCLUSION}

EL and PL at the silicon band gap energy from the MOS tunneling diodes on silicon have been studied. The radiative and nonradiative recombinations near the $\mathrm{Si} / \mathrm{SiO}_{2}$ interface are characterized by measurement of the temporal EL response, the temperature dependence of EL and PL intensities, and the voltage-dependent PL intensity. As compared to the bulk silicon, the radiative recombination coefficient in the light-emitting MOS tunneling diode is nearly one order of magnitude larger. The nonradiative SRH recombination is shown to be the dominant interband carrier recombination process. Therefore, the EL efficiency of the MOS tunneling diode can be higher if the amount of the nonradiative recombination states near the $\mathrm{Si} / \mathrm{SiO}_{2}$ interface is significantly reduced. The voltage-dependent PL intensity results from the variation of nonradiative recombination rates, which is due to the change of the Fermi level near the $\mathrm{Si} / \mathrm{SiO}_{2}$ interface with the bias voltage. The near-band-edge Fermi level for EL results in reduced and less thermally active nonradiative recombination, as compared to PL. Therefore, the EL intensity is less temperature dependent than PL. This study shows that the location of Fermi level near the $\mathrm{Si}-\mathrm{SiO}_{2}$ interface strongly influences the nonradiative SRH recombination rates and, thus, the EL and PL intensities.

\section{APPENDIX}

The physical argument for the linear approximation between the injection current density $J$ and the majority carrier density $N$ is discussed as follows. The relation between current density $J$ and carrier density $N$ is expressed as ${ }^{18}$

$$
J=T \cdot q N v,
$$

where $q$ is electronic charge, $\nu$ is the average drift velocity and $T$ is the tunneling probability across the oxide. The average drift velocity $\nu$ is saturated due to the high electrical field at the $\mathrm{Si} / \mathrm{SiO}_{2}$ interface. ${ }^{4,19}$ The tunneling probability $T$ is given by the Wentzel-Kramers-Brillouin approximation ${ }^{4}$

$$
T \approx \exp \left(-\frac{C_{T}}{\left(V_{\mathrm{ox}} / d_{\mathrm{ox}}\right)}\right),
$$

where $V_{\mathrm{ox}}$ is the voltage drop across the oxide, $d_{\mathrm{ox}}$ is the thickness of the oxide, and $C_{T}$ is a constant. The tunneling probability $T$ depends on the oxide voltage $V_{\text {ox }}$. The current density for Fowler-Nordheim tunneling $J_{\mathrm{FN}}$ and that for direct tunneling $J_{\text {Dir }}$ are derived as follows: ${ }^{4}$ 


$$
\begin{aligned}
J_{\mathrm{FN}}= & A_{\mathrm{FN}}\left(\frac{V_{\mathrm{ox}}}{d_{\mathrm{ox}}}\right)^{2} \exp \left(-\frac{B_{\mathrm{FN}}}{\left(V_{\mathrm{ox}} / d_{\mathrm{ox}}\right.}\right) \text { for } q V_{\mathrm{ox}}>\phi_{B}, \\
J_{\mathrm{Dir}}= & \frac{A_{\mathrm{FN}}}{\left(1-\sqrt{1-q V_{\mathrm{ox}} / \phi_{B}}\right)^{2}}\left(\frac{V_{\mathrm{ox}}}{d_{\mathrm{ox}}}\right)^{2} \exp \left(-\frac{B_{\mathrm{FN}}}{\left(V_{\mathrm{ox}} / d_{\mathrm{ox}}\right)}\right. \\
& \left.\times\left(1-\sqrt{1-q V_{\mathrm{ox}} / \phi_{B}}\right)^{3 / 2}\right) \text { for } q V_{\mathrm{ox}}<\phi_{B},
\end{aligned}
$$

where $A_{\mathrm{FN}}$ and $B_{\mathrm{FN}}$ are constants and $\phi_{B}$ is the barrier height at the $\mathrm{Si} / \mathrm{SiO}_{2}$ interface. The values of $A_{\mathrm{FN}}$ and $B_{\mathrm{FN}}$ are given by ${ }^{4}$

$$
\begin{aligned}
& A_{\mathrm{FN}}=1.57 \times 10^{-6} A / \mathrm{V}^{2}, \\
& B_{\mathrm{FN}}=2.57 \times 10^{8} \mathrm{~V} / \mathrm{cm}^{2} .
\end{aligned}
$$

The total applied voltage $V$ across the whole MOS tunneling diode is expressed as

$$
V=V_{\mathrm{ox}}+I \cdot R+\phi_{s}=V_{\mathrm{ox}}+A_{G} \cdot J \cdot R+\phi_{s},
$$

where $I$ is the total injection current, $R$ is the resistance of the silicon substrate, and $A_{G}=0.0154 \mathrm{~cm}^{2}$ is the gate electrode area. Furthermore, $J$ is the current density given by Eqs. (A3a) or (A3b), depending on either Fowler-Nordheim tunneling or direct tunneling, $\phi_{S}$ is the surface potential at the $\mathrm{Si} / \mathrm{SiO}_{2}$ interface and is almost a constant when the silicon surface is strongly accumulated. The resistance $R$ is approximated by $15 \Omega$ for the $n$-type silicon substrate with resistivity of $1-10 \Omega \mathrm{cm}$ and $500 \mu \mathrm{m}$ thickness. Figure 10 illustrates the relation between the oxide voltage $V_{\text {ox }}$ and the total applied voltage $V$, which is obtained by using Eqs. (A3)-(A5) for $d_{\mathrm{ox}}=30 \AA$. When the total applied voltage $V$ is greater than $7.5 \mathrm{~V}$, the increase of the oxide voltage $V_{\text {ox }}$ becomes saturated. The saturation of the oxide voltage $V_{\text {ox }}$ is due to the large voltage drop on the silicon substrate at high injection current. According to Eq. (A2), the tunneling probability $T$ will approach constant when the total applied voltage is increased beyond $7.5 \mathrm{~V}$. As a result, the expression in Eq. (A1) shows that the current density $J$ is linearly proportional to the carrier density $N$ at high applied voltages.

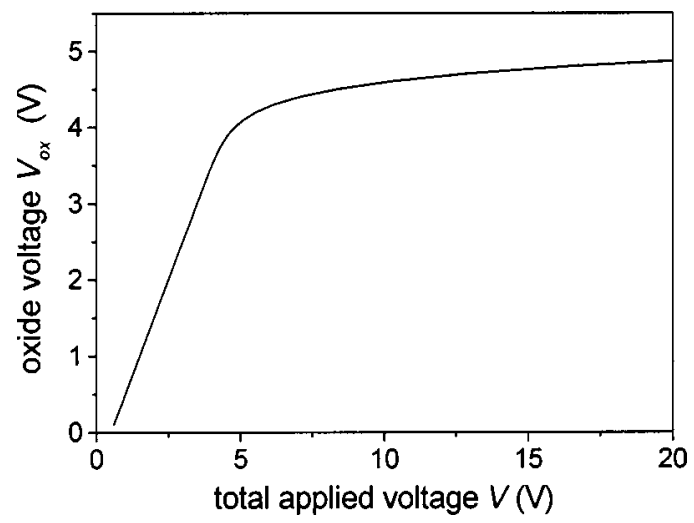

FIG. 10. Calculated oxide voltage vs total applied voltage.

\section{ACKNOWLEDGMENT}

This work was supported by the Academia Sinica, Taiwan.

${ }^{1}$ E. J. Lerner, IBM Research, Number 4 (1999).

${ }^{2}$ T. Hori, Gate Dielectrics and MOS ULSIs: Principles, Technologies, and Applications (Springer, Berlin, 1997).

${ }^{3}$ E. H. Nicollian and J. R. Brews, MOS Physics and Technology (Wiley, New York, 1982).

${ }^{4}$ D. K. Schroder, Semiconductor Material and Device Characterization, 2nd ed. (Wiley, New York, 1998).

${ }^{5}$ K. J. Yang and C. Hu, IEEE Trans. Electron Devices 46, 1500 (1999).

${ }^{6}$ C. W. Liu, M.-J. Chen, I. C. Lin, M. H. Lee, and C.-F. Lin, Appl. Phys. Lett. 77, 1111 (2000).

${ }^{7}$ M. J. Chen, E. Z. Liang, S. W. Chang, and C. F. Lin, J. Appl. Phys. 90, 789 (2001).

${ }^{8}$ M. J. Chen, C. F. Lin, M. H. Lee, S. T. Chang, and C. W. Liu, Appl. Phys. Lett. 79, 2264 (2001).

${ }^{9}$ C. W. Liu, M. H. Lee, M. J. Chen, I. C. Lin, and C. F. Lin, Appl. Phys. Lett. 76, 1516 (2000).

${ }^{10}$ C. F. Lin, C. W. Liu, M. J. Chen, M. H. Lee, and I. C. Lin, J. Appl. Phys. 87, 8793 (2000).

${ }^{11}$ C. W. Liu, M. H. Lee, M. J. Chen, C.-F. Lin, and M. Y. Chern, IEEE Electron Device Lett. 21, 601 (2000).

${ }^{12}$ J. I. Pankove, Optical Processes in Semiconductors (Dover, New York, 1971).

${ }^{13}$ E. H. Bogardus and H. B. Bebb, Phys. Rev. 176, 993 (1968).

${ }^{14}$ D. Bimberg, M. Sondergeld, and E. Grobe, Phys. Rev. B 4, 3451 (1971).

${ }^{15}$ W. Ruhle, W. Schmid, R. Meck, N. Stath, J. U. Fischbach, I. Strottner, K. W. Benz, and M. Pilkuhn, Phys. Rev. B 18, 7022 (1978).

${ }^{16}$ D. F. Blossey and P. Handler, in Semiconductor and Semimetals, edited by R. K. Willardson and A. C. Beer, (Academic, New York, 1972), Vol. 9, pp. 257-314.

${ }^{17}$ I. A. Merkulov and V. I. Perel, Phys. Lett. A 45, 83 (1973).

${ }^{18}$ J. G. Simmons, J. Appl. Phys. 34, 1793 (1963).

${ }^{19}$ S. M. Sze, Physics of Semiconductor Devices, 2nd ed. (Wiley, New York, 1981). 